\title{
PRÁTICAS DE AUDITORIA, SISTEMAS DE AVALIAÇÃO E VALORES
}

\section{AUDITING PRACTICES, EVALUATION SYSTEMS AND VALUES}

Simone Magalhães Brito*

Patrice Schuch ${ }^{* *}$

No contexto onde transparência e accountability se tornaram os valores centrais das formas de governo, as práticas de auditoria, bem como os sistemas de avaliação, monitoramento e certificação, seriam seus principais instrumentos. A potencialização das contradições num "mundo de risco" ampliou a necessidade de sistemas técnicos e legais que produzam segurança e controle, abrindo caminho para promessas de medição científica e ranqueamento dos fatos mundanos.

Nesse sentido, é possível aceitar que o diagnóstico de Michael Power (1994) acerca de uma "explosão de auditoria", surgida no contexto das reestruturações organizacionais do Reino Unido ("e sua missão liberal") no final do século XX, vai ganhando contornos locais e se tornando uma realidade mais próxima. Segundo Power, a auditoria seria uma "forma peculiar de alquimia que, ao transformar os auditados em auditáveis, produz um conforto regulatório" (POWER, 1994, p. 39) e, quando propriamente dimensionada, teria capacidade de "produzir qualidade" e não apenas avaliá-la.

É interessante perceber que essa 'alquimia peculiar' responde à necessidade de controlar os riscos e problemas da agência (derivados da assimetria de informação) por meio da promessa ou garantia de um conjunto de valores: independência, eficiência, racionalidade e visibilidade (idem). $\mathrm{O}$ avanço das formas de controle e vigilância também traz consigo um novo mundo de virtudes.

À Sociologia e Antropologia cabe refletir sobre as formas do avanço dessa alquimia, como se dá sua incorporação e, especialmente, as formas diferenciais que assume nas múltiplas modernidades. Como esse horizonte de valores é incorporado ou modifica sensibilidades? Como essas formas de valoração do bem são adotadas e

\footnotetext{
* Professora Associada do Departamento de Ciências Sociais e do Programa de Pós-Graduação em Sociologia da Universidade Federal da Paraíba (UFPB), Brasil. E-mail: simonebrito@hotmail.com

** Professora adjunta do Departamento de Antropologia, Programa de Pós-graduação em Antropologia e Programa de Pós-graduação em Políticas Públicas da UFRS. E-mail: patrice.schuch@gmail.com
} 
postas em prática? E, especialmente: como é possível que um conjunto de práticas oriundas da contabilidade tenha assumido ares de valor universal?

Para responder a essa última questão, o caminho já estabelecido nas Ciências Sociais, por meio das lógicas de racionalização e burocratização, continua sendo importante, mas precisa ainda construir uma melhor compreensão da experiência moral. Em afinidade com uma estética kafkiana sobre a forma como a racionalidade sistematicamente produz violência, diversas vertentes e projetos críticos não só incorporaram os sentidos morais de asfixia e dominação à própria descrição do sistema, como também tornaram essa forma moral a descrição por excelência das lógicas de poder. Não temos o menor interesse em minimizar a percepção da crescente heteronomia na organização do Estado. Mas, ao contrário, é do próprio interesse da crítica perceber as sutilezas da dominação e, especialmente, como as novas promessas de organização, transparentes e virtuosas, produzem novas tecnologias de controle. Destituir a lógica administrativa dos seus processos de engendramento da moral não apenas desconsidera os desenvolvimentos da burocracia e suas reformas, como também enfraquece a explicação do aprofundamento e continuidade das práticas regulatórias que constituem e projetam o Estado.

Desse modo, entendemos que investigações em Antropologia e Sociologia da moral confrontam a percepção do Estado como forma unificada e fetichizada, revelando sua natureza enquanto um objeto cultural e apontando para uma "economia moral" que tenta compreender o lugar dos valores e afetos no ordenamento de grupos, populações (FASSIN, 2015). Segundo Fassin, essa perspectiva de estudo do Estado baseada na interação entre seus agentes tem forma “indutiva, micropolítica, 'from below' (idem, p. 10). Além de perceber o Estado incorporado em práticas, lugares e linguagens, essa perspectiva também indica a necessidade de abandonar a ideia de que as recentes reformas e mudanças no Estado produziram o enfraquecimento das formas de regulação que constituem o Estado Moderno (DAS; POOLE, 2004).

O debate sobre o lugar dos sistemas de auditoria e responsabilização na construção de novas formas de governança hegemônica é um ótimo exemplo do estabelecimento dessa perspectiva (STRATHERN, 2000; SHORE, 2009; KIPNIS, 2008; SHORE and WRIGHT, 2015). O termo "cultura de auditoria" foi construído por sociólogos e antropólogos (SHORE, 2009, p. 4) para descrever uma "condição" que estava transformando a organização do sistema universitário britânico:

Uma condição moldada pelo uso de técnicas e princípios modernos de auditoria financeira, mas em contextos muito distantes do mundo da contabilidade financeira. Em outras palavras, ela se refere a contextos nos quais as técnicas e valores de prestação de contas (accountancy) tornaram-se um princípio organizador central na governança e no gerenciamento de conduta humana - e os novos tipos de relações, hábitos e práticas que isso está criando (SHORE, 2009, p. 4)

Esse autor insiste no modo como as formas supostamente neutras da auditoria transformam as maneiras como as pessoas se percebem e se relacionam, uma vez que 
são incentivadas a se compararem e medirem, situando suas capacidades pessoais em escalas de desempenho produzidas por especialistas externos. As críticas à ascensão dessa cultura se baseiam na sua natureza "colonizadora" de valores e práticas e no modo como limitam a autonomia e a criatividade (idem.).

Numa perspectiva já bem estabelecida na literatura antropológica, destacase como a sistemática de auditorias ganha cada vez mais o papel de uma tecnologia disciplinar (STRATHERN, 2000; Shore, 2009), uma parte do amplo conjunto de práticas utilizadas pelo Estado para tentar gerenciar ou pacificar populações. No entanto, não há um consenso sobre uma perspectiva de base foucaultiana que entenderia as práticas de auditoria e os sistemas de avaliação como forma da governamentalidade liberal. Por exemplo, Kpinis (2008), a partir de uma variedade de casos de auditoria na China, onde se insiste que os valores da auditoria seriam valores socialistas, chama a atenção para a necessidade de suspender a pressuposição de que todas as formas de auditoria e sistemas de avaliação produzam "regimes de verdade" liberais, dando mais ênfase às técnicas de manipulação de realidades locais e enfatizando a comparação com sociedades diversas e os múltiplos Estados sendo projetados.

Numa definição que se tornou extremamente relevante para o campo, Marilyn Strathern (2000, p. 1) afirma que o mundo da auditoria é o lugar onde “o financeiro e o moral" se encontram. Aqui, gostaríamos de aproximar esse tensionamento de valores com a imagem de Michael Power (1994, p. 39) de que as práticas de auditoria apresentam um "sentido peculiar de alquimia". A procura por uma panaceia universal capaz de curar os muitos males, inclusive morais, orientou a alquimia e é revelador que seja usado para descrever a produção de sentidos no mundo das auditorias numa promessa de que, enfim, não haverá mais contradições entre finanças e moralidade. Finalmente é possível pensar na constituição de um imperativo moral perfeitamente adequado às necessidades do sistema. Não é possível afirmar que as técnicas de auditoria tenham essa capacidade, mas é relevante compreender os desejos e utopias animando os jogos de avaliação e ranqueamento.

Contudo, enquanto uma promessa de ordenação do mundo, os valores que organizam auditorias e transparência não podem ser dissociados do fundamento do projeto do "Esclarecimento", em que a imagem da iluminação em oposição às sombras da selvageria, superstição e mistério alimentou cruzadas civilizadoras e fortaleceu o imperialismo (COMAROFF e COMAROFF, 2003). É bastante tentador aproximar Adorno e Horkheimer (1985) e os Comaroff para dizer que a fundamentação moral das práticas de auditoria seria apenas uma atualização ou reforço dos ideais civilizadores e apresentar a figura dos auditores e avaliadores como Ulisses contemporâneos (tentando tornar os outros "accountable"), numa "dialética do esclarecimento" atualizada numa situação em que o aumento da informação e controle fortalece interesses corporativos e expansão das formas de vigilância. Porém, mesmo que essa filosofia crítica do mundo da auditoria, sistemas de controle e avaliação, aponte um caminho importante de entendimento da economia política contemporânea, carece ainda dos elementos essenciais da economia moral que organiza o Estado e, especialmente, de uma compreensão dessas transformações através do cotidiano dos sistemas de avaliação e das rotinas de produção da dominação (GUPTA, 2012). 
Assim, o estudo das práticas de auditoria e sistemas de avaliação requer compreender que:

[os] novos sistemas de auditoria não são, como alegam, apenas práticas neutras e politicamente inocentes destinadas a promover "transparência" ou eficiência. Ao contrário, são tecnologias disciplinares - ou "técnicas do self" - destinadas a instilar novas normas de conduta na força de trabalho (SHORE, 2009, p.34).

No entanto, para a construção de uma Sociologia e Antropologia da vida moral, é necessário que essas formas sejam compreendidas a partir de seus contextos. Auditar e tornar-se "auditável" implicam um amplo jogo de interações a partir dos quais novos valores são definidos e, por conseguinte, as teorias capazes de compreender esse processo precisam ser construídas empiricamente.

Simone Brito, por exemplo, em pesquisas de campo realizadas com auditores de diferentes órgãos de controle, presente neste dossiê, encontrou auditores que rejeitam o próprio termo "cultura de auditoria" porque, segundos eles, não há nada parecido com esse nível de controle no caso brasileiro. Estaríamos "muito atrás". Isso não significa que devemos tomar suas palavras como o diagnóstico do caso brasileiro, mas, de maneira mais simples, entender que esses sistemas e tecnologias se desenvolvem em campos de disputas variadas e que, por exemplo, nossa nostalgia por mais controle seja um horizonte mais importante para moldar a organização dos valores do que o problema do respeito aos rituais internacionais de certificação.

Nessa perspectiva, fica claro, como afirma Bourdieu (2014), que o "oficial" é resultado de um conjunto de disputas. No caso brasileiro, diferenças entre os sistemas federal e estadual, o objeto das políticas, carreiras dos auditores, níveis de participação da sociedade civil, por exemplo, geram disputas capazes de modificar os sentidos de auditorias e avaliações. Apesar de suas diferenças, o sentido geral da perspectiva bourdieusiana, especialmente em sua crítica às perspectivas idealistas e legalistas, pode ser relacionado com tendências já indicadas que analisam o Estado e as tecnologias de governo por meio das disputas e projeções de valores morais (SCOTT,1998; COMAROFF e COMAROFF, 2003; FASSIN, 2012; GUPTA, 2012). O que está em jogo é compreender como as ficções e valores, as projeções e imaginação de uma ordem justa, se tornam efetivas. Assim, as compreensões sociológica e antropológica se movem para uma problematização do poder estatal e das burocracias em que se torna necessário conhecer as práticas de validação de seu discurso, a construção de redes de apoio ao seu estabelecimento e a geração de gramáticas morais que pretendem organizar a vida pública, bem como os modos pelos quais movimentos e práticas de sua contestação e/ou resistência são efetivados.

Desse modo, os artigos apresentados neste dossiê se articulam na tentativa de responder a esse debate, tendo como elemento central o cotidiano da produção de valores e sentidos de justiça em diferentes perspectivas.

Em “O novo marco regulatório das organizações da sociedade civil e uma nova agenda de pesquisa sobre as relações entre os órgãos de controle, as OSCs e os gestores públicos”, Rogério Medeiros e Nínive Machado analisam, a partir de um histórico da constituição do Marco Regulatório das Organizações da Sociedade Civil 
(MROSc), as tensões e as disputas na operacionalização do novo código jurídico que ordena as relações entre o Estado e as Organizações da Sociedade Civil no Brasil. Ao refletir sobre a relação entre Estado e Sociedade Civil, a partir dos processos e práticas de contratualização e prestação de contas, o artigo de Medeiros e Machado nos permite perceber o papel efetivo dos discursos morais e das práticas de controle no cotidiano do Estado. Uma vez que o problema da corrupção se estabelece como um horizonte moral de regulação das ações do Estado, as interações entre Estado e Sociedade Civil são reorganizadas. Contudo, a pesquisa demonstra como o estabelecimento da nova regulamentação que busca evitar as formas de corrupção não é um simples ajuste das técnicas empregadas, mas uma rearticulação entre valores e práticas. Em diferentes momentos do processo de "seleção, contratação e avaliação das parcerias", a nova regulamentação desafia as práticas estabelecidas dos funcionários do Estado e gera um novo ambiente de negociação em que valores morais e políticos se sobrepõem às formas tidas como puramente técnicas ou legais.

Também tensionado os ideais técnicos e os valores morais, no artigo "Para além da técnica: experiência moral e combate à corrupção em processos de auditoria pública", Simone Brito apresenta a experiência de auditores num Tribunal de Contas Estadual. $\mathrm{O}$ artigo busca reconstruir a experiência de engajamento nas atividades de combate à corrupção e demonstrar como a constituição dos sentidos da auditoria das contas públicas projetam um ideal ético que seria capaz de superar e evitar as diversas políticas. Sua proposta é que a dimensão do "moralismo", normalmente rejeitada nas análises políticas dos problemas do Estado, seja incorporada pela Sociologia da Moral como um de seus mecanismos de produção de valores na vida política.

Em "Lei sob a Norma: o saber jurídico e os processos normalizadores nas Auditorias de Justiça Militar Estadual", as práticas de auditoria são entendidas como uma forma normalizadora da ação de policiais e bombeiros militares. Por meio de uma análise foucaultiana, Fábio França, Anderson Duarte e Geni Francinelli buscam demonstrar a centralidade dada aos crimes que ferem os valores e a ética militar e, consequentemente, como as auditorias militares seriam máquinas de produção e ordenação da vida moral castrense. O histórico das auditorias militares no Brasil revela que o seu lugar na produção de regimes de verdade não é recente, mas foi longamente gestado como um elemento central para a manutenção das virtudes projetadas pela vida militar.

Assim, essa perspectiva da Sociologia da Moral aproxima as auditorias militares do disciplinamento militar, demonstrando que as práticas de auditoria não são rituais de exceção, mas uma forma cotidiana de manutenção da ordem.

Seguindo a perspectiva de compreensão das auditorias como forma de ordenação do cotidiano, temos um outro artigo sobre o lugar das auditorias na vida das Polícias Militares: "O Testemunho sobre policiais: formas de construção de verdade na Auditoria da Justiça Militar do Estado do Rio de Janeiro" de Sabrina Souza da Silva. Por meio de observação e de entrevistas com diversos agentes envolvidos em processo de auditoria militar, a autora analisa o processo de construção da verdade jurídica a partir de dois casos específicos. O ritual de produção do inquérito é utilizado como o fio condutor para a compreensão da 
mediação entre os saberes práticos e os ideais normativos, de modo que a auditoria militar é marcada por processo de tradução da realidade em formas jurídicas. Com base nas experiências analisadas, demonstra-se como os rituais de auditoria, ainda que se estabeleçam como uma promessa de justiça e isenção, terminam por "corroborar a obediência à hierarquia como a principal forma de controle sobre o policial".

As duas discussões sobre os processos de auditoria militar ajudam a deslocar a compreensão comum dos processos de auditoria como um conjunto de regras impessoais e revelam sua composição como uma forma agonística em que indivíduos em diferentes posições na hierarquia social disputam os sentidos de justiça. Em contraposição aos ideais de isenção e valores técnicos que definem a autoimagem dos auditores do tribunal de contas (Brito, nessa coletânea), os dois últimos trabalhos referidos acima permitem entrever a posição dos "auditados" e, especialmente, os efeitos dessa posição na construção e ordenamento dos valores. Desse modo, as práticas de auditoria se mostram como um ambiente privilegiado para compreensão das disputas de valor que organizam o Estado, ainda que possamos percebê-las nos diversos mecanismos que organizam as ações estatais (como será demonstrado no primeiro trabalho deste dossiê).

Do ponto de vista da Sociologia e Antropologia da Moral, a importância dessas perspectivas está em revelar as práticas que atualizam e produzem o normativo. Nesse sentido, o nosso último artigo representa um caminho importante na realização dessa abordagem prática do problema dos valores chamando a atenção para a necessidade de ir além da ideia de "construção social dos valores", à medida que se incorpora à análise do problema da articulação e da interpretação de experiências traumáticas.

Em “"Eu não sabia que era crime’: inarticulação linguística do sofrimento em situações de violência intrafamiliar”, Andressa L. M. Lima e Carlos Eduardo Freitas analisam o problema do sofrimento moral e as experiências de reorientação de valores a partir de dois casos de violência intrafamiliar. A incapacidade das duas mulheres entrevistadas (Valéria e Lena) de, quando crianças, compreenderem a natureza das violências às quais eram submetidas chama a atenção para o fato de que a experiência moral do sofrimento é também um processo ativo aberto a novas articulações e interpretações de valores. Essa perspectiva permite abandonar os sentidos naturalizadores da experiência moral sem recair em formas relativistas e ainda reinserir a dimensão dinâmica nas experiências morais. $\mathrm{O}$ diálogo pode ser estabelecido com as discussões anteriores na medida em que os autores fornecem uma importante perspectiva para a compreensão da "gênese e adesão a valores" constitutivos de gramáticas morais que pretendem organizar a vida pública.

Tomando o conjunto destes artigos, há uma variedade de leituras possíveis sobre o problema dos valores nas perspectivas sociológica e antropológica. No entanto, destacamos mais uma vez que essa reunião buscou pensar as rotinas de produção dos sentidos de justo e correto através do tensionamento entre hierarquias e ordens estabelecidas e as formas de interpretação e reflexividade que caracterizam a vida moral, principalmente, o que caracteriza a discussão geral é um desafio à crença na possibilidade de técnicas de organização social neutras e apolíticas. Essa 
perspectiva se torna necessária quando se observa, em diversos lugares, as tentativas de substituição do debate político por um conjunto de técnicas administrativas, em que se busca que as diferentes projeções do Estado ideal sejam substituídas por uma crença em rotinas e valores universalmente desejáveis. Esperamos, assim, que as discussões contribuam para a compreensão dos meios envolvidos na construção de novas éticas em relação à formação de sujeitos e da cidadania no Brasil e seus sentidos e/ou contestações.

\section{Referências}

ADORNO, T.; HORKHEIMER, M. A dialética do Esclarecimento: Fragmentos filosóficos. Rio de Janeiro: Jorge Zahar, 1985.

BOURDIEU, P. Razões práticas: Sobre a Teoria da ação. Campinas: Papirus, 2011.

BOURDIEU, P. Sobre o Estado: Cursos no College de France (1989-92). São Paulo: Cia. das Letras, 2014.

COMAROFF, J; COMAROFF, J. Ethnography and the Historical imagination. Boulder, San Francisco: Westview Press, 1992.

COMAROFF, J; COMAROFF, J. Transparent Fictions; or, the Conspiracies of a liberal imagination: an afterword. In: WEST, H. G; SANDERS, T. (Ed.) Transparency and Conspiracy: Ethnographies of suspicion in the New World Order. Durham and London: Duke University Press, 2003.

DAS, V.; POOLE, D. (Ed). Anthropology in the margins of State. Oxford, New Tork: Oxford University Press, 2004.

FASSIN, D. (Ed.). A companion to moral anthropology. Oxford: Wiley-Blackwell, 2012.

FASSIN, D. (et al.). At the heart of States. The moral world of institutions. London: Pluto Press, 2015.

FERGUSON, J.; GUPTA, A. Spatializing States: Toward an Ethnography of Neoliberal Governmentality. American Ethnologist. Vol. 29, No. 4, p. 981-1002, 2002

FOUCAULT, M. Microfísica do poder. Rio de Janeiro e São Paulo: Paz e Terra, 2016.

GUPTA, A. Red tape: bureaucracy, structural violence, and poverty in India. Durham: Duke University Press, 2012.

HULL, S. M. Government of Paper: The Materiality of Bureaucracy in Urban Pakistan. Oakland: University of California Press, 2012

KIPNIS, A. Audit cultures: Neoliberal governmentality, socialist legacy, or technologies of governing?

American Ethnologist, Vol. 35, No. 2, pp. 275-289, 2008

POWER, M. The audit explosion. London: Demos, No. 7, 1994.

POWER, M. The audit society: rituals of verification. Oxford: Oxford University Press, 1997.

SCOTT, J. C. Seeing like a State: How certain schemes to improve the human condition have failed. New Haven and London: Yale University Press, 1998.

SHARMA, A.; GUPTA, A.(Ed.) The Anthropology of the State: a reader. Malden, Oxford, Victoria: Blackwell Publishing, 2006.

SHORE, C. Cultura de auditoria e governança iliberal: universidades e a política da responsabilização. Mediações. v. 14, n. 1. Londrina. 2009

SHORE, C.; WRIGHT, S. Governing by numbers: audit culture, rankings and the new world order. Social Anthropology/Anthropologie Sociale, 2015, 23, 1 22-28.

STRATHERN, M. Audit cultures: anthropological studies in accountability, ethics, and the academy. London: Routledge. 2000 